\title{
Drift and Diffusion in Periodically Driven Renewal Processes
}

\author{
T. Prager*and L. Schimansky-Geier \\ Institute of Physics, Humboldt-University at Berlin, \\ Newtonstr. 15, D-12489 Berlin, Germany
}

22nd September 2018

\begin{abstract}
We consider the drift and diffusion properties of periodically driven renewal processes. These processes are defined by a periodically time dependent waiting time distribution, which governs the interval between subsequent events. We show that the growth of the cumulants of the number of events is asymptotically periodic and develop a theory which relates these periodic growth coefficients to the waiting time distribution defining the periodic renewal process. The first two coefficients, which are the mean frequency and effective diffusion coefficient of the number of events are considered in greater detail. They may be used to quantify stochastic synchronization.
\end{abstract}

\section{Introduction}

Many dynamical processes in physics, biology and chemistry, although being inherently continuous, can be reduced to a series of discrete events without losing much information. Often this reduced description in terms of discrete events has the additional property, that time intervals between subsequent events are statistically independent. Such processes are called renewal processes [1. They are fully described by a waiting time distribution $w(\tau)$, which governs the statistical properties of the time intervals $\tau$ between two subsequent events. In the simplest case the probability per unit time, i.e. the rate, for an event to occur is independent on the time elapsed since the last event. This subclass of renewal processes is called Markovian, because the event number of such a process as a function of time is a Markovian stochastic process. If driven externally, the rate can

*e-mail tobias@physik.hu-berlin.de 
be temporally modulated becoming thus a function of the absolute time $t$, but still remains independent on the waiting time $\tau$ elapsed since the previous event.

In the general case, the probability per unit time that an event will happen is not independent of the time already elapsed since the last event. Then a non exponentially distributed waiting time between subsequent events is observed which distinguishes these general renewal processes from discrete Markovian processes.

The concepts of renewal processes have been successfully applied to random sequences of spiking events in the dynamics of neurons [3, 4, 5], to random walks in a tilted periodic potential [6] and to the failure times of biological and technical machineries [7]. In these models the processes which generate the single events consist of a series of several Markovian steps or possess a priori complicated waiting time densities. For example, the generation of a new spike requires excitation of the voltage variable over a threshold value which is followed by the spiking and refractory time where neurons are unable to produce new spikes. Also the ionic transport through pores involves several steps as diffusional motion followed by escapes over potential barriers. Molecular motors walk along microtubuli where sequences of different configurational changes of the proteins realize one forward step.

There is a long history of studies of stationary renewal processes [1], i.e. renewal processes having waiting time densities $w(\tau)$, which do not depend on the absolute time $t$. Also stationary situations with multiple events and different stationary waiting time statistics were studied. Quantities of interest like the mean event number in a certain period of time or the event number diffusion are known in terms of the waiting time distribution, because a fully developed theory exists for these time homogeneous processes 1. [7]

In connection with stochastic resonance (SR) the interest for non stationary but periodic processes has grown [8, 9, 10]. In particular, Markovian periodically driven models have been investigated [11]. The two state Markovian theory with non stationary rates describes successfully the dynamic behavior in bistable situations as shown in many different studies in various fields of science. However, other systems like excitable dynamics as used for example to describe the spiking mechanism in neuronal systems can no longer be approximated by a simple discrete Markovian description. If such systems are periodically driven, as is for example the case in neurons which respond to periodically varying inputs, more general concepts are needed to describe their behavior. In this paper we study the general situation of non stationary but periodic renewal processes with arbitrary waiting time distributions $w(\tau, t)$. The periodicity is reflected by a periodic dependence of the waiting time distribution on the absolute time $t$ of the 
previous event.

There exist different possibilities to quantify the periodicity of a periodic stochastic process, or, if one considers this periodicity as induced by an external periodic signal, the quality of the response of the system to this periodic signal. On the one hand, spectral based measures like the spectral power amplification and the signal to noise ratio have been frequently employed (see [10] and references therein). These spectral based response measures where also considered in the context of periodically driven renewal processes in [16, 17] and for a special discrete state model for excitable dynamics in [18. To this end the sequence of events has to be somehow mapped onto a stochastic process, e.g. by considering a sequence of delta peaks located at the event times or by assigning alternatingly after each event different values to the process. Another possibility to characterize periodicity of the process is to consider the drift and diffusion properties of the number of events. The evolution of the number of events in time can be characterized by two quantities, the mean frequency of events and the effective diffusion coefficient, which describes, how the variance of the number of events grows in time and thus characterizes the regularity of the process. The lower this effective diffusion coefficient for a fixed mean frequency, the more regular, i.e. periodic, is the system's dynamics. Both quantities together may therefore serve as a measure of periodicity of the process and thus, if this periodicity stems form the influence of a periodic input, as a measure of stochastic synchronization between the driving signal and the system dynamics [14, 12, 15, 13, 21.

In this paper we present a method to calculate the mean frequency and the effective diffusion coefficient of a periodically driven renewal process in terms of its periodically time dependent waiting time distribution $w(\tau, t)$. After having derived the general concepts and results in sections 2 to 5 . we consider two simple situations, namely general but undriven renewal processes and periodically modulated rate process, for which an explicit evaluation of the general results is possible and agrees with the known results. Finally in section 7 and 8 we numerically evaluate our theory for a toy model where the intervals between subsequent events are governed by a a fixed but periodically varying waiting time followed by a rate process with constant rate. The results agree with simulations of the underlying periodic renewal process. Many of the calculations are summarized in a series of appendices.

\section{Periodically driven renewal processes}

A periodically driven renewal process is a sequence of events occurring at times $\ldots, t_{i}, t_{i+1}, t_{i+2}, \ldots$. The intervals between two subsequent events 
are governed by the waiting time distribution $w(\tau, t)$. The first argument $\tau$ represents the waiting time whereas the second argument $t$ denotes the absolute time at which the last event happened. Therefore, $w\left(\tau, t_{i}\right) d \tau$ is the probability that the event $i+1$ happens in the time interval $\left(t_{i}+\tau, t_{i}+\tau+d \tau\right]$ if event $i$ have been happened at time $t_{i}$. The dependence of the waiting time distribution $w(\tau, t)$ on time $t$ is due to the periodic driving and thus periodic with the period $\mathcal{T}=2 \pi / \Omega$ of the signal. Although, in contrast to an ordinary renewal process, the intervals between subsequent events are now correlated, it is still only the time of the previous event which governs the statistics of the following event, which justifies to stick to the term renewal. Normalization holds at arbitrary time $t$

$$
\int_{0}^{\infty} d \tau w(\tau, t)=1
$$

A lot of information about these processes is contained in the random number of events $N_{t_{0}, t}$ which take place in the interval $\left(t_{0}, t\right]$. For example stochastic synchronization to the periodic driving can be characterized as an integer relation between driving frequency and the frequency of events and at the same time, a decrease in the effective diffusion coefficient of the events, i.e. a more regular (periodic) behavior.

We will evaluate the mean frequency and effective diffusion coefficient based on the periodically time dependent waiting time distribution $w(\tau, t)$, which defines the periodically driven renewal process. To this end we consider more generally the $n$th cumulants $K_{t_{0}, t}^{(n)}$ of the event number $N_{t_{0}, t}$ and define their increment per time

$$
\kappa_{t_{0}, t}^{(n)}:=\lim _{\Delta t \rightarrow 0} \frac{K_{t_{0}, t+\Delta t}^{(n)}-K_{t_{0}, t}^{(n)}}{\Delta t}=\frac{d}{d t} K_{t_{0}, t}^{(n)} .
$$

The first of these coefficients is the mean frequency while the second is the effective diffusion coefficient. We show that asymptotically

$$
\kappa^{(n)}(t):=\lim _{t_{0} \rightarrow-\infty} \kappa_{t_{0}, t}^{(n)}
$$

become periodic functions of time with the period of the external driving.

To this end we introduce following [19, 2] the generating functional $L_{t_{0}, t}[v]$ of the considered driven renewal process as

$$
L_{t_{0}, t}[v]=\left\langle\prod_{i=1}^{N_{t_{0}, t}}\left(1+v\left(t_{i}\right)\right)\right\rangle
$$

where $t_{i}$ are the times of the events in the interval $\left(t_{0}, t\right]$. Then the $n$th moment $M_{t_{0}, t}^{(n)}$ of event number $N_{t_{0}, t}$ is given by

$$
M_{t_{0}, t}^{(n)}:=\left\langle N_{t_{0}, t}^{n}\right\rangle=\left.\frac{\partial^{n}}{\partial u^{n}} L_{t_{0}, t}\left[e^{u}-1\right]\right|_{u=0} .
$$


The generating functional $L_{t_{0}, t}$ can be expressed in terms of the distribution functions $f_{s}\left(t_{1}, \ldots, t_{s}\right)$, which govern the probability

$$
d P=f_{s}\left(t_{1}, \ldots, t_{s}\right) d t_{1} \ldots d t_{s}
$$

to find one event in each of the intervals $\left(t_{i}, t_{i}+d t_{i}\right), i=1, \ldots, s$ regardless of how many events are outside these intervals, as [19, 2 ,

$L_{t_{0}, t}[v]=1+\sum_{s=1}^{\infty} \int_{t_{0}}^{t} d \tau_{1} \int_{t_{0}}^{\tau_{1}} d \tau_{2} \ldots \int_{t_{0}}^{\tau_{s-1}} d \tau_{s} f_{s}\left(\tau_{1}, \tau_{2}, \ldots, \tau_{s}\right) v\left(\tau_{1}\right) \ldots v\left(\tau_{s}\right)$.

The generating functional can also be expressed in terms of the correlation functions $g_{s}\left(t_{1}, \ldots, t_{s}\right)$ as

$$
\begin{aligned}
& L_{t_{0}, t}[v]=\exp [ \\
& \left.\quad \sum_{s=1}^{\infty} \int_{t_{0}}^{t} d \tau_{1} \int_{t_{0}}^{\tau_{1}} d \tau_{2} \ldots \int_{t_{0}}^{\tau_{s-1}} d \tau_{s} g_{s}\left(\tau_{1}, \tau_{2}, \ldots, \tau_{s}\right) v\left(\tau_{1}\right) \ldots v\left(\tau_{s}\right)\right] .
\end{aligned}
$$

Eq. (5) together with eq. (4) define the correlation functions in terms of the distribution functions.

According to eq. (5) the moments eq. (3) can be expressed as

$$
M_{t_{0}, t}^{(n)}=\left.\frac{\partial^{n}}{\partial u^{n}} \exp \left[\sum_{s=1}^{\infty} G_{s}\left(t_{0}, t\right)\left(e^{u}-1\right)^{s}\right]\right|_{u=0}
$$

where

$$
G_{s}\left(t_{0}, t\right):=\int_{t_{0}}^{t} d \tau_{1} \int_{t_{0}}^{\tau_{1}} d \tau_{2} \ldots \int_{t_{0}}^{\tau_{s-1}} d \tau_{s} g_{s}\left(\tau_{1}, \tau_{2}, \ldots, \tau_{s}\right)
$$

From formula (6) we can evaluate the corresponding cumulants $K_{t_{1}, t_{2}}^{(n)}$ as (see appendix B)

$$
K_{t_{0}, t}^{(n)}=\left.\frac{\partial^{n}}{\partial u^{n}} \sum_{s=1}^{\infty} G_{s}\left(t_{0}, t\right)\left(e^{u}-1\right)^{s}\right|_{u=0} .
$$

As the considered renewal processes are periodic in time with period $\mathcal{T}$, the distribution functions and therefore also the correlation functions are likewise periodic in time,

$$
g_{s}\left(\tau_{1}, \ldots, \tau_{s}\right)=g_{s}\left(\tau_{1}+\mathcal{T}, \ldots, \tau_{s}+\mathcal{T}\right) .
$$


Then the time derivative of the function $G_{s}\left(t_{0}, t\right)$ yields

$$
\begin{aligned}
\frac{d}{d t} G_{s}\left(t_{0}, t\right) & =\int_{t_{0}}^{t} d \tau_{2} \ldots \int_{t_{0}}^{\tau_{s-1}} d \tau_{s} g_{s}\left(t, \tau_{2}, \ldots, \tau_{s}\right) \\
& =\frac{1}{(s-1) !} \int_{t_{0}}^{t} d \tau_{2} \ldots \int_{t_{0}}^{t} d \tau_{s} g_{s}\left(t, \tau_{2}, \ldots, \tau_{s}\right) \\
& =\frac{1}{(s-1) !} \int_{0}^{t-t_{0}} d \tau_{2} \ldots \int_{0}^{t-t_{0}} d \tau_{s} g_{s}\left(t, t-\tau_{2}, \ldots, t-\tau_{s}\right)
\end{aligned}
$$

which can be expressed in the asymptotic limit as

$$
\lim _{t_{0} \rightarrow-\infty} \frac{d}{d t} G_{s}\left(t_{0}, t\right)=\frac{1}{(s-1) !} \int_{0}^{\infty} d \tau_{2} \ldots \int_{0}^{\infty} d \tau_{s} g_{s}\left(t, t-\tau_{2}, \ldots, t-\tau_{s}\right) .
$$

To ensure that this limit exists, we additionally suppose that $g_{s}\left(\tau_{1}, \ldots, \tau_{s}\right)$ decreases sufficiently fast to zero for any pair of time difference $\left|\tau_{i}-\tau_{j}\right| \rightarrow$ $\infty$. In [2] this property is called cluster property, while for stationary systems this property is called ergodicity.

According to eq. (8) the asymptotic time derivative eq. (9) is a periodic function in $t$ and thus (cf. eq. (7)) the coefficients

$$
\kappa^{(n)}(t)=\lim _{t_{0} \rightarrow-\infty} \frac{d}{d t} K_{t_{0}, t}^{(n)} .
$$

are periodic in time, as well.

The time dependent waiting time distribution can be expressed in terms of the distribution functions as 2

$$
w(\tau, t)=f_{1}(t+\tau)+\sum_{s=1}^{\infty} \frac{(-1)^{s}}{s !} \int_{t}^{t+\tau} d t_{1} \ldots d t_{s} f_{s+1}\left(t+\tau, t_{1}, \ldots, t_{s}\right) .
$$

However, we are faced with the inverse problem, namely to express the distribution functions $f_{s}$ or likewise the correlation functions $g_{s}$ in terms of the waiting time distribution $w(\tau, t)$ in order to finally evaluate the coefficients $\kappa^{(n)}(t)$ according to eqs. (10) and (7). Such a relation is not known to the authors and even if it exists, the explicit evaluation of the infinite sum in eq. (17) will be challenging. Thus to obtain the $\kappa^{(n)}(t)$ in terms of the time dependent waiting time distribution $w(\tau, t)$ we have to adopt a different approach.

\section{The microscopic master equation}

Let us consider the probabilities $p_{k}(t)$ to have had $k$ events up to time $t$. Furthermore let $j_{k}(t)$ be the probability flux from state $k$ to state $k+1$, 
i.e. the probability per time that the $k+1^{\text {st }}$ event happens at time $t$. This probability obeys the continuity equation

$$
\frac{d}{d t} p_{k}(t)=j_{k-1}(t)-j_{k}(t)
$$

If we further assume as initial condition that event 1 happened at time $t_{0}$, i.e. $j_{0}(t)=\delta\left(t-t_{0}\right)$, the relation between the probability fluxes of the renewal process can be expressed by the "microscopic" dynamics as (for the undriven case see e.g. [20])

$$
j_{k}(t)=\int_{t_{0}}^{t} d t^{\prime} j_{k-1}\left(t^{\prime}\right) w\left(t-t^{\prime}, t^{\prime}\right), \quad k \geq 1 .
$$

Using this relation one readily obtains from the continuity equation (11)

$$
p_{k}(t)=\int_{t_{0}}^{t} d t^{\prime} j_{k-1}\left(t^{\prime}\right) z\left(t-t^{\prime}, t^{\prime}\right), \quad k \geq 1
$$

where $z(\tau, t)=1-\int_{0}^{\tau} d \tau^{\prime} w\left(\tau^{\prime}, t\right)$ is the probability to wait longer than $\tau$ until the next event, if the last event happened at $t$. In case of a Markovian renewal process with time dependent rate $\gamma(t)$ the probability flux $j_{k}$ is related to the probability $p_{k}$ by $j_{k}(t)=\gamma(t) p_{k}(t)$. Thus in this case the dynamics can be completely expressed in terms of the probabilities $p_{k}$. In the general case however we need a formulation in terms of $p_{k}$ and $j_{k}$ as expressed in eqs. (13) and (12).

The moments of the number of events $\tilde{N}_{t_{0}, t}$ in the interval $\left(t_{0}, t\right]$ can be expressed in terms of the $p_{k}$ as

$$
\tilde{M}_{t_{0}, t}^{(n)}=\sum_{k=0}^{\infty} k^{n} p_{k}(t)
$$

Note that the moments $\tilde{M}_{t_{0}, t}^{(n)}$ differ from the moments defined by eq. (3) since the corresponding event number $\tilde{N}_{t_{0}, t}$ is conditioned on having had an event at time $t_{0}$ in contrast to $N_{t_{0}, t}$. However the asymptotic behavior of both families of moments agrees.

In principle one can calculate the cumulants from the moments eq. (14) to obtain eventually the coefficients $\kappa^{(n)}(t)$ according to eq. (10). However, in practice this is not feasible in general, as one has to calculate an infinite sum over the $p_{k}(t)$ where each $p_{k}$, according to eqs. (13) and (12), is a $k$-fold integral involving the waiting time distributions $w(\tau, t)$ and the corresponding survival probabilities $z(\tau, t)$. 


\section{Embedding the discrete dynamics into a con- tinuous one}

To find a simpler relation between the periodic coefficients $\kappa^{(n)}(t)$ and the time dependent waiting time $w(\tau, t)$, which governs the microscopic dynamics, we construct a continuous embedding in the asymptotic limit $t_{0} \rightarrow-\infty$. Consider the envelope $\mathcal{P}(x, t)$ of the discrete probabilities $p_{k}(t)$ as a probability distribution on a continuous state space. Thus, respecting the normalization we adopt the relation (cf. Fig. 1)

$$
p_{k}(t)=\int_{k-\frac{1}{2}}^{k+\frac{1}{2}} d x \mathcal{P}(x, t) .
$$

Assuming that the variation of $\mathcal{P}(x, t)$ within an interval $\left(k-\frac{1}{2}, k+\frac{1}{2}\right)$

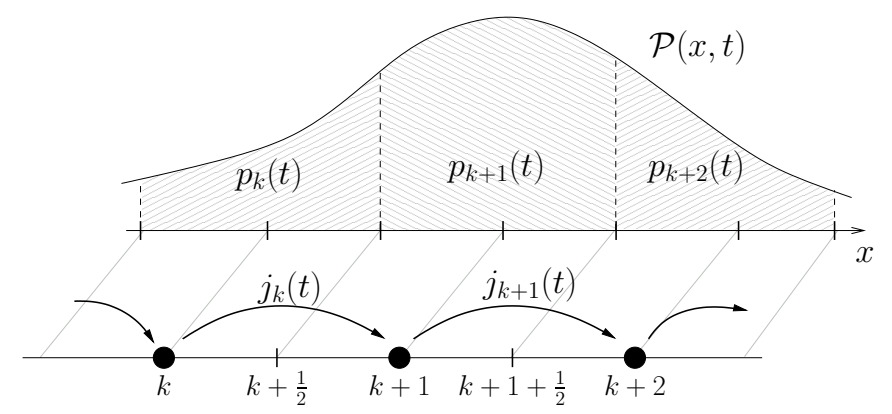

Figure 1: Schematic view of the discrete event dynamics $p_{k}(t)$ and the continuous description in terms of $x$.

becomes arbitrary small for large $t$ the difference between the moments and therefore also the cumulants of both the discrete and the continuous process tends to zero in this asymptotic limit. Accordingly, also the change in time of the cumulants of the continuous process have to agree with the corresponding quantities $\kappa^{(n)}(t)$ of the discrete process.

One possible choice to formulate a dynamics of the continuous envelope $\mathcal{P}(x, t)$ is a Kramers-Moyal equation. This choice renders the underlying stochastic process $x(t)$ Markovian. To ensure the equality of the increments of the cumulants, the coefficients of the Kramers-Moyal expansion have to coincide with the coefficients $\kappa^{(n)}(t)$ of the renewal process (cf. appendix C] [19]), i.e.

$$
\frac{\partial}{\partial t} \mathcal{P}(x, t)=\sum_{n=1}^{\infty} \frac{(-1)^{n}}{n !} \kappa^{(n)}(t) \frac{\partial^{n}}{\partial x^{n}} \mathcal{P}(x, t)
$$


The probability current $j_{k}(t)$ in the discrete system is correspondingly related to the probability current of the continuous envelope description $\mathcal{J}(x, t)$. According to the relation between the discrete and continuous probability eq. (15), the discrete probability current $j_{k}(t)$ from $k$ to $k+1$ is equal to the continuous probability current $\mathcal{J}(x, t)$ at $x=k+\frac{1}{2}$,

$$
j_{k}(t)=\mathcal{J}\left(k+\frac{1}{2}, t\right)
$$

The continuous probability current $\mathcal{J}(x, t)$ is related to the probability distribution $\mathcal{P}(x, t)$ by the continuity equation

$$
\frac{\partial}{\partial t} \mathcal{P}(x, t)=-\frac{\partial}{\partial x} \mathcal{J}(x, t) .
$$

and therefore according to eq. (16)

$$
\mathcal{J}(x, t)=-\sum_{n=1}^{\infty} \frac{(-1)^{n}}{n !} \kappa^{(n)}(t) \frac{\partial^{n-1}}{\partial x^{n-1}} \mathcal{P}(x, t) .
$$

Thus from eq. (17) we deduce

$$
j_{k}(t)=-\left.\sum_{n=1}^{\infty} \frac{(-1)^{n}}{n !} \kappa^{(n)}(t) \frac{\partial^{n-1}}{\partial x^{n-1}} \mathcal{P}(x, t)\right|_{x=k+\frac{1}{2}}
$$

Finally we want to mention that the more general embedding of the discrete process

$p_{k}(t)=\int_{k-1+l}^{k+l} d x \mathcal{P}(x, t) \quad$ and $\quad j_{k}(t)=\mathcal{J}(k+l, t), \quad l$ arbitrary.

leads to the same results as the embedding chosen in eqs. (15) and (17), which corresponds to choosing $l=\frac{1}{2}$ in eq. (21).

\section{The asymptotic drift and the diffusion coef- ficient}

Having fixed the relation between the probabilities and probability fluxes of the discrete renewal process and the continuous embedding, it is now possible to relate the coefficients $\kappa^{(n)}(t)$ appearing in the continuous description (16) to the waiting time distribution $w(\tau, t)$ of the renewal process, involved in the microscopic dynamics (12) and (13). As we are considering 
the asymptotic behavior we have to pass to the asymptotic limit in eqs. (13) and (12) by shifting the initial time $t_{0} \rightarrow-\infty$. This results in

$$
p_{k}(t)=\int_{0}^{\infty} d \tau j_{k-1}(t-\tau) z(\tau, t-\tau)
$$

and

$$
j_{k}(t)=\int_{0}^{\infty} d \tau j_{k-1}(t-\tau) w(\tau, t-\tau)
$$

Inserting eqs. (15) and (20) into the above eq. (22) we end up with

$$
\begin{aligned}
\int_{-\frac{1}{2}}^{\frac{1}{2}} d \Delta x \mathcal{P}(x-\Delta x, t)= & -\int_{0}^{\infty} d \tau z(\tau, t-\tau) \\
& \sum_{n=1}^{\infty} \frac{(-1)^{n}}{n !} \kappa^{(n)}(t-\tau) \frac{\partial^{n-1}}{\partial x^{n-1}} \mathcal{P}\left(x-\frac{1}{2}, t-\tau\right)
\end{aligned}
$$

with $x=k$. The probability $\mathcal{P}(x-\Delta x, t-\tau)$ can be expressed in terms of the probability $\mathcal{P}(x, t)$ and its derivatives $\frac{\partial^{m}}{\partial x^{m}} \mathcal{P}(x, t)$ by performing a Taylor expansion of $\mathcal{P}(x-\Delta x, t-\tau)$ around $x, t$ and converting the time derivatives to derivatives with respect to the state $x$ using the Kramers-

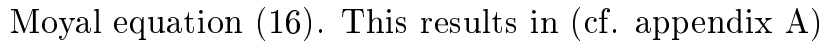

$$
\mathcal{P}(x-\Delta x, t-\tau)=\mathcal{P}(x, t)+c_{t}^{(1)}(t-\tau, \Delta x) \frac{\partial}{\partial x} \mathcal{P}(x, t)+O(2)
$$

with

$$
c_{t}^{(1)}(t-\tau, \Delta x)=\int_{0}^{\tau} d \tau^{\prime} \kappa^{(1)}\left(t-\tau^{\prime}\right)-\Delta x .
$$

Above $O(2)$ denotes terms proportional to $\frac{\partial^{m}}{\partial x^{m}} \mathcal{P}(x, t), m \geq 2$.

Denoting by $L$ the characteristic length scale over which the probability distribution $\mathcal{P}(x, t)$ varies, we may introduce the new variable $\tilde{x}=\frac{x}{L}$. Then

$$
\frac{\partial^{m}}{\partial x^{m}} \mathcal{P}(x, t)=\left.\frac{1}{L^{m}} \frac{\partial^{m}}{\partial \tilde{x}^{m}} \mathcal{P}(\tilde{x}, t)\right|_{\tilde{x}=\frac{x}{L}}
$$

Due to the dispersion of the probability distribution $\mathcal{P}(x, t)$ the characteristic length $L$ asymptotically goes to infinity.

Equating the coefficients of $\mathcal{P}(x, t)$ and $\frac{\partial}{\partial x} \mathcal{P}(x, t)$ on both sides of eq. (24), or equivalently, considering the 0 th and 1 st order in $L$, we end up 
with

$$
\begin{aligned}
& \int_{0}^{\infty} d \tau \kappa^{(1)}(t-\tau) z(\tau, t-\tau)=1 \\
& \frac{1}{2} \int_{0}^{\infty} d \tau \kappa^{(2)}(t-\tau) z(\tau, t-\tau)= \\
& \int_{0}^{\infty} d \tau \kappa^{(1)}(t-\tau)\left[\int_{0}^{\tau} d \tau^{\prime} \kappa^{(1)}\left(t-\tau^{\prime}\right)-\frac{1}{2}\right] z(\tau, t-\tau) .
\end{aligned}
$$

Eq. (26) can be further simplified using eq. (25), which leads to

$$
\begin{aligned}
& \int_{0}^{\infty} d \tau \kappa^{(2)}(t-\tau) z(\tau, t-\tau)= \\
& 2 \int_{0}^{\infty} d \tau \kappa^{(1)}(t-\tau) \int_{0}^{\tau} d \tau^{\prime} \kappa^{(1)}\left(t-\tau^{\prime}\right) z(\tau, t-\tau)-1
\end{aligned}
$$

These two expressions, relating the asymptotic drift and diffusion properties of a periodically driven renewal process as expressed by $\kappa^{(1)}(t)$ and $\kappa^{(2)}(t)$ to its microscopic properties defined by $z(\tau, t)$ present the cornerstone result of our paper. Equations which govern the higher cumulant growth coefficients $\kappa^{(n)}(t), n \geq 3$ can also be derived using this method by evaluating the coefficients of higher order derivatives of $\mathcal{P}(x, t)$ (for $\kappa^{(3)}(t)$ see appendix D].

In a former work [21] we investigated stochastic synchronization in a non Markovian two state model for excitable systems. The result presented in this paper for the mean velocity and effective diffusion coefficient can be shown to be equivalent to the results presented here (see appendix $\mathbb{E}$ ).

Often one is not directly interested in the number of events but in some quantity proportional to the number of events, like a phase, which increases by $\mathfrak{L}=2 \pi$ for each event, or a position which increases by some length $\mathfrak{L}=l$ if we consider a unidirectional random walk with step-length $l$ whose steps are governed by the described renewal dynamics. In these cases the $i$ th cumulant growth coefficient $\kappa^{(i)}(t)$ is scaled by $\mathfrak{L}^{i}$. For $\kappa^{(1)}(t)$ and $\kappa^{(2)}(t)$ this is achieved multiplying the constant term 1 on the right hand side of eqs. (25), and (27) by $\mathfrak{L}$ or $\mathfrak{L}^{2}$ respectively.

Finally one may ask, why it is justified to prescribe a continuous Markovian envelope dynamics to an inherently non Markovian discrete process. The obvious idea, that the non Markovian nature of the discrete process is rendered Markovian by being mapped onto an extended continuous state space is misleading. The point is, that the continuous Markovian process $x(t)$ as described by eq. (16) is not an envelope dynamics of the full discrete non Markovian process, but it only covers the asymptotic behavior of the non Markovian process, i.e the regime, where the discrete process has forgotten its initial preparation. 


\section{Comparison with known results for undriven renewal processes and periodically driven rate processes}

Let us evaluate expressions (25) and (26) for an undriven renewal process, i.e. $z(\tau, t) \equiv z(\tau)$. Then it follows that $\kappa^{(1)}(t)$ is constant and eq. 25) leads to

$$
\kappa^{(1)}(t)=\frac{\mathfrak{L}}{\langle T\rangle}
$$

with

$$
\left\langle T^{n}\right\rangle:=\int_{0}^{\infty} d \tau \tau^{n} w(\tau) .
$$

To derive this result we have used the fact that

$$
\int_{0}^{\infty} d \tau \tau^{n} z(\tau)=\int_{0}^{\infty} d \tau \frac{\tau^{n+1}}{n+1} w(\tau)=\frac{1}{n+1}\left\langle T^{n+1}\right\rangle
$$

which holds if $z(\tau)$ decreases sufficiently fast for $\tau \rightarrow \infty$. Accordingly eq. (27) gives

$$
\kappa^{(2)}(t)=\mathfrak{L}^{2} \frac{\left\langle T^{2}\right\rangle-\langle T\rangle^{2}}{\langle T\rangle^{3}}
$$

which agrees with the known results for stationary renewal processes [1]. The corresponding expression for $\kappa^{(3)}(t)$ is presented in appendix D

Next we consider a periodically driven Markov process, i.e.

$$
w(\tau, t)=\gamma(t+\tau) \exp \left(-\int_{t}^{t+\tau} d \tau^{\prime} \gamma\left(\tau^{\prime}\right)\right)
$$

and

$$
z(\tau, t)=\exp \left(-\int_{t}^{t+\tau} d \tau^{\prime} \gamma\left(\tau^{\prime}\right)\right)
$$

Then it can be easily shown that eq. (25) is solved by

$$
\kappa^{(1)}(t)=\mathfrak{L} \gamma(t) .
$$

The first term on the right hand side og eq. (27) can also be simplified in this case using integration by parts to give $\mathfrak{L}^{2}$. Therefore $\kappa^{(2)}(t)$ is governed by

$$
\int_{0}^{\infty} d \tau \kappa^{(2)}(t-\tau) z(\tau, t-\tau)=\mathfrak{L}^{2}
$$


which is solved by

$$
\kappa^{(2)}(t)=\mathfrak{L}^{2} \gamma(t)
$$

For more complicated processes with general time dependent waiting time distributions eqs. (25) and (27) can only be solved numerically for the periodic solution.

\section{Numerical solution in Fourier space}

As eqs. (25) and (26) cannot be solved analytically for arbitrary waiting time distributions $w(\tau, t-\tau)$ and corresponding survival probabilities $z(\tau, t-\tau)$ one has to resort to numerical methods. To this end we perform a Fourier expansion of the periodic function $\kappa^{(1)}(t)$ and analogously for $\kappa^{(2)}(t)$

$\kappa^{(1)}(t)=\sum_{k=-\infty}^{\infty} \kappa_{k}^{(1)} \exp (i k \Omega t), \quad \kappa_{k}^{(1)}=\frac{1}{\mathcal{T}} \int_{0}^{\mathcal{T}} d t \kappa^{(1)}(t) \exp (-i k \Omega t)$,

where $\Omega=2 \pi / \mathcal{T}$ is the frequency of the external driving. We further expand the survival probability $z(\tau, t)$ with respect to the second periodic argument as

$z(\tau, t)=\sum_{k=-\infty}^{\infty} z_{k}(\tau) \exp (i k \Omega t) \quad z_{k}(\tau)=\frac{1}{\mathcal{T}} \int_{0}^{\mathcal{T}} d t z(\tau, t) \exp (-i k \Omega t)$

Abbreviating

$$
z_{k, l}=\int_{0}^{\infty} d \tau z_{k}(\tau) \exp (-i l \Omega \tau), \quad h_{k, l}=\int_{0}^{\infty} d \tau \tau z_{k}(\tau) \exp (-i l \Omega \tau)
$$

eq. (25) can be written as

$$
\sum_{k=-\infty}^{\infty} \kappa_{k}^{(1)} z_{m-k, m}=\mathfrak{L} \delta_{m, 0}, \quad m=-\infty, \ldots, \infty
$$

while eq. (27) reads

$$
\begin{aligned}
& \sum_{k=-\infty}^{\infty} \kappa_{k}^{(2)} z_{m-k, m}=2 \sum_{k=-\infty}^{\infty} \\
& \left.\quad\left[\sum_{\substack{l=-\infty, l \neq 0 \\
-\mathfrak{L}^{2} \delta_{m, 0} .}}^{\infty} \frac{\kappa_{l}^{(1)} \kappa_{k}^{(1)}}{i l \Omega}\left(z_{m-k-l, m-l}-z_{m-k-l, m}\right)\right]+\kappa_{0}^{(1)} \kappa_{k}^{(1)} h_{m-k, m}\right] \\
& \quad
\end{aligned}
$$


The corresponding equation for the third coefficient $\kappa^{(3)}(t)$ in Fourier space is presented in appendix D.

These infinite dimensional inhomogeneous linear equations can then be numerically solved, after being truncated to a finite dimensional system.

\section{A simple example-comparison between the- ory and simulations}

Consider the toy renewal process, where the time between subsequent events is composed of a fixed waiting time, which depends on the signal phase of the previous event and a rate process with rate $\gamma$. The waiting time distribution is thus given by

$$
w(\tau, t)=\theta(\tau-T(t)) \gamma e^{-\gamma(\tau-T(t))} .
$$

Suppose further that the fixed waiting time is either $T_{0}$ or $T_{1}$ depending on whether the signal phase of the previous event was within $[0, \pi)$ or $[\pi, 2 \pi)$, i..e

$$
T(t)=\left\{\begin{array}{lll}
T_{0} & \text { if } \Omega t & \bmod 2 \pi \in[0, \pi) \\
T_{1} & \text { if } \Omega t & \bmod 2 \pi \in[\pi, 2 \pi)
\end{array}\right.
$$

A sketch of this system is shown in Fig. 2 while corresponding waiting time distribution is plotted in Fig. 3

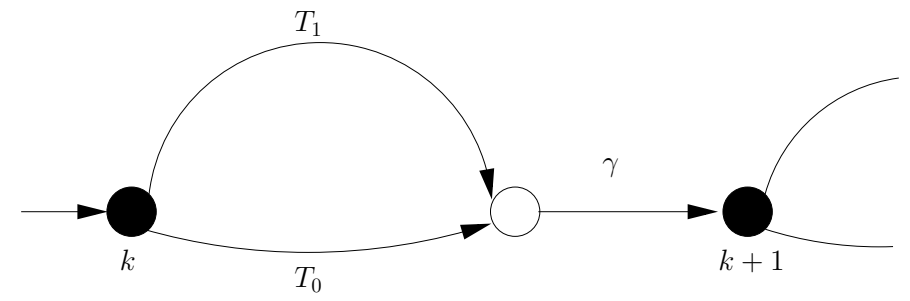

Figure 2: Depending on whether the periodic signal is in the first or second half period the system either waits the fixed time $T_{0}$ or $T_{1}$. In both cases the system waits an additional exponentially with rate $\gamma$ distributed time.

The corresponding Fourier coefficients $z_{k, l}$ and $h_{k, l}$ as presented in eqs. (29) can be analytically evaluated for this waiting time distribution, however the final results, being too long and at the same time yielding not much information, will not be presented here. Having evaluated these Fourier coefficients, we calculated the mean frequency and the effective diffusion 


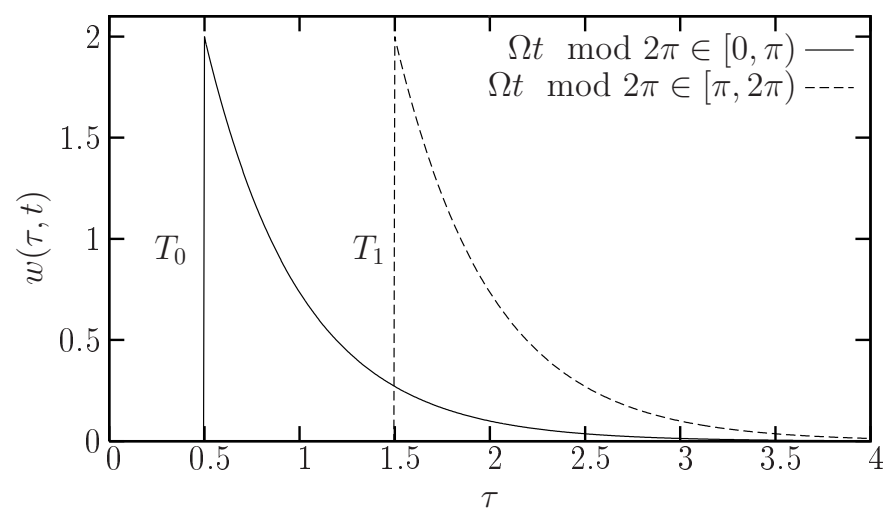

Figure 3: Waiting time distribution of the toy model eq. (32) with dichotomic fixed waiting time from eq. (32) for $T_{0}=0.5, T_{1}=1.5$ and $\gamma=2$. Depending on the signal phase of the event, the system waits either a long (dashed line) or a short time(solid line) plus an exponentially distributed time until the next event.

coefficient according to eqs. (30) and (31) using LAPACK to solve these linear equations. The results are compared to simulations of the renewal process in Figs 4 and 5 showing perfect agreement.

The mean velocity $\bar{\kappa}^{(1)}=\frac{1}{\mathcal{T}} \int_{0}^{\mathcal{T}} d t \kappa^{(1)}(t)$ and effective diffusion coefficient $\bar{\kappa}^{(2)}=\frac{1}{\mathcal{T}} \int_{0}^{\mathcal{T}} d t \kappa^{(2)}(t)$ can be used to characterize stochastic synchronization of the process to the periodic signal. These synchronization regions are defined by a rational relation between system frequency and signal frequency and a minimum of the effective diffusion coefficient. Although this model shows minima of the effective diffusion coefficient as a function of the driving frequency Fig. 4. these minima do not correspond to frequency synchronization as the system frequency and the signal frequency are not proportional. This stands in contrast to a similar model with a fixed waiting time and a dichotomically periodically modulated rate, used to describe periodically driven excitable systems [21]. In this model we found several different $n: m$ synchronization regions. Also the full periodically time dependent coefficients $\kappa^{(1)}(t)$ and $\kappa^{(2)}(t)$ as determined by our theory (25) and (27) agree with results taken from simulations of the underlying renewal process Fig. 5. Interestingly the effective diffusion coefficient becomes negative for some values of the signal phase. However this does not imply that the periodic driving can be used to concentrate an ensemble of these systems as the period averaged effective diffusion coefficient $\bar{\kappa}^{(2)}$ is always positive. 


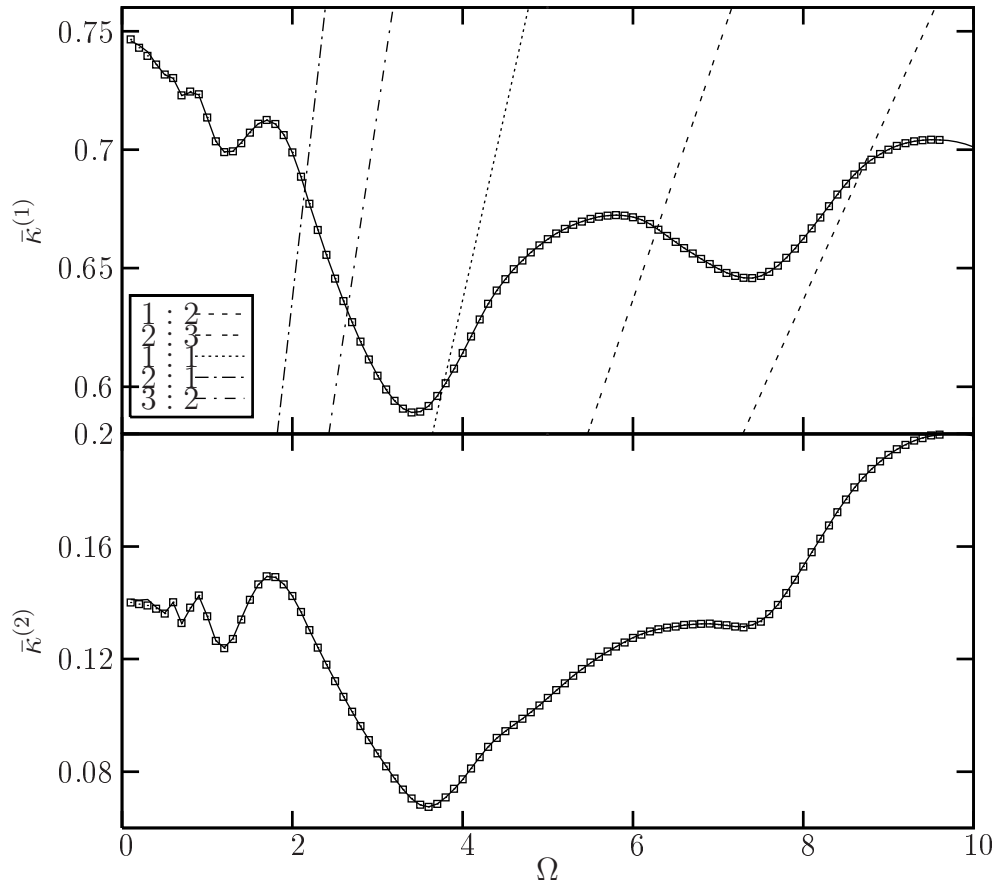

Figure 4: Comparison of the period averaged mean frequency $\bar{\kappa}^{(1)}=$ $\frac{1}{\mathcal{T}} \int_{0}^{\mathcal{T}} d t \kappa^{(1)}(t)$ and the period averaged effective diffusion coefficients $\bar{\kappa}^{(2)}=$ $\frac{1}{\mathcal{T}} \int_{0}^{\mathcal{T}} d t \kappa^{(2)}(t)$ where $\mathcal{T}=2 \pi / \Omega$ is the period of the signal, for the toy model eq. (32) with $T_{0}=0.5, T_{1}=1.5$ and $\gamma=2$. The solid lines are results of the theory eqs. (25) and (27), numerically evaluated according to eqs. (30) and (31) truncated to 40 coefficients, while the symbols are obtained from direct simulations of the driven renewal process. The straight lines in the upper plot indicate $n: m$ relations between system frequency and signal frequency, i.e. frequency locking. Clearly the system does not show this behavior. 


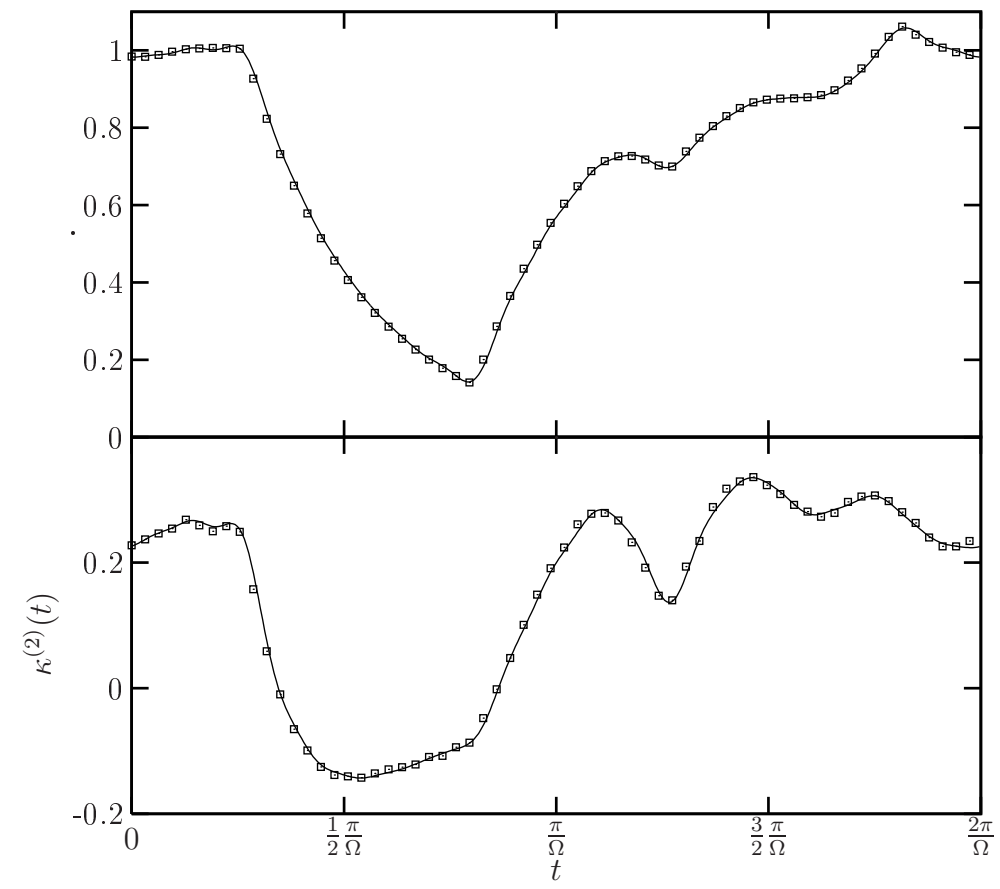

Figure 5: Comparison of the mean frequency $\kappa^{(1)}(t)$ and the effective diffusion coefficients $\kappa^{(2)}(t)$ for the toy model eq. (32) with $T_{0}=0.5$, $T_{1}=1.5$ and $\gamma=2$ and $\Omega=1.7$. The solid lines are results of the theory eqs. (25) and (27), numerically evaluated according to eqs. (30) and (31) truncated to 20 coefficients, while the symbols are obtained from direct simulations of the driven renewal process. 


\section{Conclusion}

We have considered the drift and diffusion behavior of periodic renewal processes and presented a general theory to express the mean frequency and effective diffusion constant and also higher order cumulant growth coefficients in terms of the underlying time dependent waiting time distribution. The results of this approach where analytically shown to coincide with known results in the undriven case. For the periodically driven situation we confirmed the results of the theory by numerical investigations. We also showed agreement with a different approach for a more restricted class of renewal processes presented in [21]. The mean frequency and effective diffusion coefficient may be used to quantify stochastic synchronization. Taking into account the large amount of systems whose dynamics can be modeled as renewal processes, we anticipate a widespread applicability of our results, ranging from synchronization in neurons to the investigation of transport properties in molecular motors.

\section{A Expansion of the probability density gov- erned by a Kramers-Moyal equation}

Our aim is to express the phase distribution $\mathcal{P}(x-\Delta x, t-\tau)$ in terms of $\mathcal{P}(x, t)$ and its derivatives with respect to $x, \partial^{n} / \partial x^{n} \mathcal{P}(x, t)$. To this end we start by expanding $\mathcal{P}(x-\Delta x, t-\tau))$ in a Taylor series around $x$ and $t$,

$$
\mathcal{P}(x-\Delta x, t-\tau)=\sum_{n=0}^{\infty} \sum_{m=0}^{\infty} \frac{(-\Delta x)^{n}(-\tau)^{m}}{n ! m !} \frac{\partial^{n+m}}{\partial x^{n} \partial t^{m}} \mathcal{P}(x, t)
$$

To process the time derivatives we use the Kramers-Moyal equation (16) taking care of the explicit time dependence of $\kappa^{(i)}(t)$ which leads to

$$
\begin{aligned}
& \mathcal{P}(x-\Delta x, t-\tau)=\mathcal{P}(x, t)-\left[\Delta x+\sum_{m=1}^{\infty} \frac{(-\tau)^{m}}{m !} \frac{\partial^{m-1} \kappa^{(1)}(t)}{\partial t^{m-1}}\right] \frac{\partial}{\partial x} \mathcal{P}(x, t) \\
& +\left[\frac{\Delta x^{2}}{2}+\Delta x \sum_{m=1}^{\infty} \frac{(-\tau)^{m}}{m !} \frac{\partial^{m-1} \kappa^{(1)}(t)}{\partial t^{m-1}}+\frac{1}{2} \sum_{m=1}^{\infty} \frac{(-\tau)^{m}}{m !} \frac{\partial^{m-1} \kappa^{(2)}(t)}{\partial t^{m-1}}\right. \\
& \left.+\sum_{m=2}^{\infty} \frac{(-\tau)^{m}}{m !} \sum_{l=1}^{m-1}\left(\begin{array}{c}
m-1 \\
l
\end{array}\right) \frac{\partial^{m-1-l} \kappa^{(1)}(t)}{\partial t^{m-1-l}} \frac{\partial^{l-1} \kappa^{(1)}(t)}{\partial t^{l-1}}\right] \frac{\partial^{2}}{\partial x^{2}} \mathcal{P}(x, t)+O(3) .
\end{aligned}
$$

where $O(3)$ denotes third or higher derivatives of $\mathcal{P}(x, t)$ with respect to $x$. The sums containing the coefficients $\kappa^{(n)}(t)$ in a linear way be further 
evaluated, leading to

$$
\begin{aligned}
\sum_{m=1}^{\infty} \frac{(-\tau)^{m}}{m !} \frac{\partial^{m-1} \kappa^{(n)}(t)}{\partial t^{m-1}} & =-\sum_{m=0}^{\infty} \frac{1}{m !} \frac{\partial^{m} \kappa^{(n)}(t)}{\partial t^{m}} \int_{0}^{\tau} d \tau^{\prime}\left(-\tau^{\prime}\right)^{m} \\
& =-\int_{0}^{\tau} d \tau^{\prime} \kappa^{(n)}\left(t-\tau^{\prime}\right)
\end{aligned}
$$

The last term can be simplified to give

$$
\begin{aligned}
\sum_{m=2}^{\infty} & \frac{(-\tau)^{m}}{m !} \sum_{l=1}^{m-1}\left(\begin{array}{c}
m-1 \\
l
\end{array}\right) \frac{\partial^{m-1-l} \kappa^{(1)}(t)}{\partial t^{m-1-l}} \frac{\partial^{l-1} \kappa^{(1)}(t)}{\partial t^{l-1}} \\
& =\sum_{l=0}^{\infty} \sum_{m=0}^{\infty} \frac{(-\tau)^{m+l+2}}{(m+l+2) !}\left(\begin{array}{c}
m+l+1 \\
l+1
\end{array}\right) \frac{\partial^{m} \kappa^{(1)}(t)}{\partial t^{m}} \frac{\partial^{l} \kappa^{(1)}(t)}{\partial t^{l}} \\
& =\int_{0}^{\tau} d \tau^{\prime} \sum_{m=0}^{\infty} \frac{\left(-\tau^{\prime}\right)^{m}}{m !} \frac{\partial^{m} \kappa^{(1)}(t)}{\partial t^{m}} \int_{0}^{\tau^{\prime}} d \tau^{\prime \prime} \sum_{l=0}^{\infty} \frac{\left(-\tau^{\prime \prime}\right)^{l}}{l !} \frac{\partial^{l} \kappa^{(1)}(t)}{\partial t^{l}} \\
& =\int_{0}^{\tau} d \tau^{\prime} \kappa^{(1)}\left(t-\tau^{\prime}\right) \int_{0}^{\tau^{\prime}} d \tau^{\prime \prime} \kappa^{(1)}\left(t-\tau^{\prime \prime}\right)
\end{aligned}
$$

Thus we eventually arrive at

$$
\begin{aligned}
\mathcal{P}(x-\Delta x, t-\tau)= & \mathcal{P}(x, t)+c_{t}^{(1)}(t-\tau, \Delta x) \frac{\partial}{\partial x} \mathcal{P}(x, t) \\
& +c_{t}^{(2)}(t-\tau, \Delta x) \frac{\partial^{2}}{\partial x^{2}} \mathcal{P}(x, t)+O(3)
\end{aligned}
$$

where

$$
c_{t}^{(1)}(t-\tau, \Delta x)=\int_{0}^{\tau} d \tau^{\prime} \kappa^{(1)}\left(t-\tau^{\prime}\right)-\Delta x
$$

and

$$
\begin{aligned}
& c_{t}^{(2)}(t-\tau, \Delta x)=\frac{\Delta x^{2}}{2}-\Delta x \int_{0}^{\tau} d \tau^{\prime} \kappa^{(1)}\left(t-\tau^{\prime}\right) \\
& -\frac{1}{2} \int_{0}^{\tau} d \tau^{\prime} \kappa^{(2)}\left(t-\tau^{\prime}\right)+\int_{0}^{\tau} d \tau^{\prime} \kappa^{(1)}\left(t-\tau^{\prime}\right) \int_{0}^{\tau^{\prime}} d \tau^{\prime \prime} \kappa^{(1)}\left(t-\tau^{\prime \prime}\right)
\end{aligned}
$$




\section{B Relation between moments and cumulants of the number of events of a renewal process and its characteristic functional}

Consider the moments $M^{(n)}$ defined by (c.f. eq (6))

$$
M^{(n)}=\left.\frac{\partial^{n}}{\partial z^{n}} \exp \left[\sum_{s=1}^{\infty} G_{s}\left(e^{z}-1\right)^{s}\right]\right|_{z=0} .
$$

Generally the relation between moments and cumulants is given by

$$
\sum_{k=0}^{\infty} \frac{z^{k}}{k !} M^{(k)}=\exp \left[\sum_{k=1}^{\infty} \frac{z^{k}}{k !} K^{(k)}\right] .
$$

From the relation

$$
M^{(n)}=\left.\frac{\partial^{n}}{\partial z^{n}} \sum_{k=0}^{\infty} \frac{z^{k}}{k !} M^{(k)}\right|_{z=0}
$$

and eq. (36) we deduce (taking into account the analyticity at $z=0$ of the functions involved)

$$
\sum_{k=0}^{\infty} \frac{z^{k}}{k !} M^{(k)}=\exp \left[\sum_{s=1}^{\infty} G_{s}\left(e^{z}-1\right)^{s}\right]
$$

and thus according to eq. (37)

$$
\sum_{k=1}^{\infty} \frac{z^{k}}{k !} K^{(k)}=\sum_{s=1}^{\infty} G_{s}\left(e^{z}-1\right)^{s}
$$

With

$$
K^{(n)}=\left.\frac{\partial^{n}}{\partial z^{n}} \sum_{k=1}^{\infty} \frac{z^{k}}{k !} K^{(k)}\right|_{z=0}
$$

the final result

$$
K^{(n)}=\left.\frac{\partial^{n}}{\partial z^{n}} \sum_{s=1}^{\infty} G_{s}\left(e^{z}-1\right)^{s}\right|_{z=0}
$$

follows. 


\section{Relation between the Kramers-Moyal coef- ficient and the growth of the cumulants}

Consider the stochastic process $x(t)$ governed by

$$
\frac{\partial}{\partial t} \mathcal{P}(x, t)=\sum_{n=1}^{\infty} \frac{(-1)^{n}}{n !} \kappa^{(n)}(t) \frac{\partial^{n}}{\partial x^{n}} \mathcal{P}(x, t)
$$

We are interested in the grows of the cumulants $K^{(n)}(t)$ of $x(t)$. The moments

$$
M^{(n)}(t)=\left\langle x^{n}(t)\right\rangle=\int_{-\infty}^{\infty} d x x^{n} \mathcal{P}(x, t)
$$

obey

$$
\begin{aligned}
\frac{d}{d t} M^{(n)}(t) & =\int_{-\infty}^{\infty} d x x^{n} \frac{\partial}{\partial t} \mathcal{P}(x, t) \\
& =\sum_{j=1}^{\infty} \frac{(-1)^{j}}{j !} \kappa^{(j)}(t) \int_{-\infty}^{\infty} d x x^{n} \frac{\partial^{j}}{\partial x^{j}} \mathcal{P}(x, t)
\end{aligned}
$$

Assuming further that $\mathcal{P}(x, t)$ decreases sufficiently fast for $x \rightarrow \pm \infty$ such that

$$
\lim _{x \rightarrow \pm \infty} x^{n} \mathcal{P}(x, t)=0
$$

the above expression can be evaluated using integration by parts to give

$$
\begin{aligned}
\frac{d}{d t} M^{(n)}(t) & =\sum_{j=1}^{\infty} \frac{\kappa^{(j)}(t)}{j !} \int_{-\infty}^{\infty} d x\left[\frac{\partial^{j}}{\partial x^{j}} x^{n}\right] \mathcal{P}(x, t) \\
& =\sum_{j=1}^{n} \frac{\kappa^{(j)}(t)}{j !} \int_{-\infty}^{\infty} d x \frac{n !}{(n-j) !} x^{n-j} \mathcal{P}(x, t) \\
& =\sum_{j=1}^{n} \kappa^{(j)}(t)\left(\begin{array}{c}
n \\
j
\end{array}\right) M^{(n-j)}(t)
\end{aligned}
$$

Now the moments and cumulants are related by

$$
\sum_{k=0}^{\infty} \frac{z^{k}}{k !} M^{(k)}(t)=\exp \left[\sum_{k=1}^{\infty} \frac{z^{k}}{k !} K^{(k)}(t)\right] .
$$

and thus by differentiating this equation with respect to $t$

$$
\sum_{k=0}^{\infty} \frac{z^{k}}{k !} \frac{d}{d t} M^{(k)}(t)=\sum_{k=1}^{\infty} \frac{z^{k}}{k !} \frac{d}{d t} K^{(k)}(t) \sum_{k=0}^{\infty} \frac{z^{k}}{k !} M^{(k)}(t) .
$$


Inserting the moments dynamic eq. (39) into the left hand side of this equation, it can be easily checked that

$$
\frac{d}{d t} K^{(n)}(t)=\kappa^{(n)}(t)
$$

\section{The third cumulant growth coefficient $\kappa^{(3)}(t)$}

The third coefficient $\kappa^{(3)}(t)$ can be evaluated by equating the coefficients corresponding to the order $\frac{\partial^{2}}{\partial x^{2}} \mathcal{P}(x, t)$ in eq. (24). Inserting the expansion (33) into this equation leads to

$$
\begin{aligned}
& \frac{1}{6} \int_{0}^{\infty} d \tau \kappa^{(3)}(t-\tau) z(\tau, t-\tau)= \\
& \quad \frac{1}{2} \int_{0}^{\infty} d \tau \kappa^{(2)}(t-\tau)\left[\int_{0}^{\tau} d \tau^{\prime} \kappa^{(1)}\left(t-\tau^{\prime}\right)-\frac{1}{2}\right] z(\tau, t-\tau) \\
& \quad-\int_{0}^{\infty} d \tau \kappa^{(1)}(t-\tau)\left[\frac{1}{6}-\frac{1}{2} \int_{0}^{\tau} d \tau^{\prime} \kappa^{(1)}\left(t-\tau^{\prime}\right)-\frac{1}{2} \int_{0}^{\tau} d \tau^{\prime} \kappa^{(2)}\left(t-\tau^{\prime}\right)\right. \\
& \left.\quad+\int_{0}^{\tau} d \tau^{\prime} \kappa^{(1)}\left(t-\tau^{\prime}\right) \int_{0}^{\tau^{\prime}} d \tau^{\prime \prime} \kappa^{(1)}\left(t-\tau^{\prime \prime}\right)\right] z(\tau, t-\tau)
\end{aligned}
$$

or using eqs. (25) and (27)

$$
\begin{gathered}
\int_{0}^{\infty} d \tau \kappa^{(3)}(t-\tau) z(\tau, t-\tau)=\int_{0}^{\infty} d \tau z(\tau, t-\tau) \\
{\left[3 \kappa^{(2)}(t-\tau) \int_{0}^{\tau} d \tau^{\prime} \kappa^{(1)}\left(t-\tau^{\prime}\right)+3 \kappa^{(1)}(t-\tau) \int_{0}^{\tau} d \tau^{\prime} \kappa^{(2)}\left(t-\tau^{\prime}\right)\right.} \\
\left.-6 \kappa^{(1)}(t-\tau) \int_{0}^{\tau} d \tau^{\prime} \kappa^{(1)}\left(t-\tau^{\prime}\right) \int_{0}^{\tau^{\prime}} d \tau^{\prime \prime} \kappa^{(1)}\left(t-\tau^{\prime \prime}\right)\right]+1
\end{gathered}
$$

For an undriven renewal process eq. (43) can be directly solved, leading to the time independent coefficient

$$
\kappa^{(3)}(t)=\mathfrak{L}^{3} \frac{\langle T\rangle^{4}-3\left\langle T^{2}\right\rangle\langle T\rangle^{2}+3\left\langle T^{2}\right\rangle^{2}-\langle T\rangle\left\langle T^{3}\right\rangle}{\langle T\rangle^{5}}
$$

For a rate process with periodically modulated rate $\gamma(t)$ (43) eventually leads to

$$
\kappa^{(3)}(t)=\mathfrak{L}^{3} \gamma(t) .
$$

Generally, the periodic solution

$$
\kappa^{(3)}(t)=\sum_{k=-\infty}^{\infty} \kappa_{k}^{(3)} \exp (i k \Omega t), \quad \kappa_{k}^{(3)}=\frac{1}{\mathcal{T}} \int_{0}^{\mathcal{T}} d t \kappa^{(3)}(t) \exp (-i k \Omega t),
$$


of eq. (43) can be numerically obtained in Fourier space as a solution of the (infinite) set of linear equations

$$
\begin{aligned}
& \sum_{k=-\infty}^{\infty} \kappa_{k}^{(3)} z_{m-k, m}= \\
& 3 \sum_{k=-\infty}^{\infty}\left[\left[\sum_{l=-\infty, l \neq 0}^{\infty} \frac{\kappa_{l}^{(2)} \kappa_{k}^{(1)}+\kappa_{l}^{(1)} \kappa_{k}^{(2)}}{i l \Omega}\left(z_{m-k-l, m-l}-z_{m-k-l, m}\right)\right]\right. \\
& \left.+\left[\kappa_{0}^{(2)} \kappa_{k}^{(1)}+\kappa_{0}^{(1)} \kappa_{k}^{(2)}\right] h_{m-k, m}\right] \\
& -6 \sum_{k=-\infty}^{\infty}\left[\left[\sum_{l=-\infty, l \neq 0}^{\infty} \sum_{j=-\infty, j \neq 0,-l}^{\infty}\right.\right. \\
& \frac{\kappa_{k}^{(1)} \kappa_{l}^{(1)} \kappa_{j}^{(1)}}{j \Omega^{2}}\left(\frac{1}{l}\left(z_{m-k-l-j, m-j}-z_{m-k-l-j, m-j-l}\right)\right. \\
& \left.\left.+\frac{1}{j+l}\left(z_{m-k-l-j, m-j-l}-z_{m-k-l-j, m}\right)\right)\right] \\
& +\sum_{l=-\infty, l \neq 0}^{\infty} \frac{\kappa_{k}^{(1)} \kappa_{l}^{(1)} \kappa_{-l}^{(1)}}{l^{2} \Omega^{2}}\left(z_{m-k, m}-z_{m-k, m-l}+i l \Omega h_{m-k, m}\right) \\
& \left.+\sum_{l=-\infty, l \neq 0}^{\infty} \frac{\kappa_{k}^{(1)} \kappa_{l}^{(1)} \kappa_{0}^{(1)}}{i l \Omega}\left(h_{m-k-l, m-l}-h_{m-k-l, m}\right)+\kappa_{k}^{(1)} \kappa_{0}^{(1)} \kappa_{0}^{(1)} j_{m-k, m}\right] \\
& +\mathfrak{L}^{3} \delta_{m, 0} .
\end{aligned}
$$

where we used the Fourier decompositions of $\kappa^{(1)}$ and $\kappa^{(2)}$ according to eqs. (28) while $z_{k, l}$ and $h_{k, l}$ are defined in eq. (29) and $j_{k, l}$ is defined as

$$
j_{k, l}=\frac{1}{2} \int_{0}^{\infty} d \tau \tau^{2} z_{k}(\tau) \exp (-i l \Omega \tau) .
$$

For the toy model introduced in section 8 we have evaluated the period average of $\kappa^{(3)}(t)$ both according to the theory, eqs. (43) and (44) and from direct simulations of the driven renewal process. Both results agree very well (Fig. (6)), thus confirming our theory.

\section{E Equivalence with the two state model for excitable systems}

In a former work 21] we investigated stochastic synchronization in a non Markovian two state model for excitable systems. Such an excitable dynamics can be approximated by two steps, namely an excitation from the 


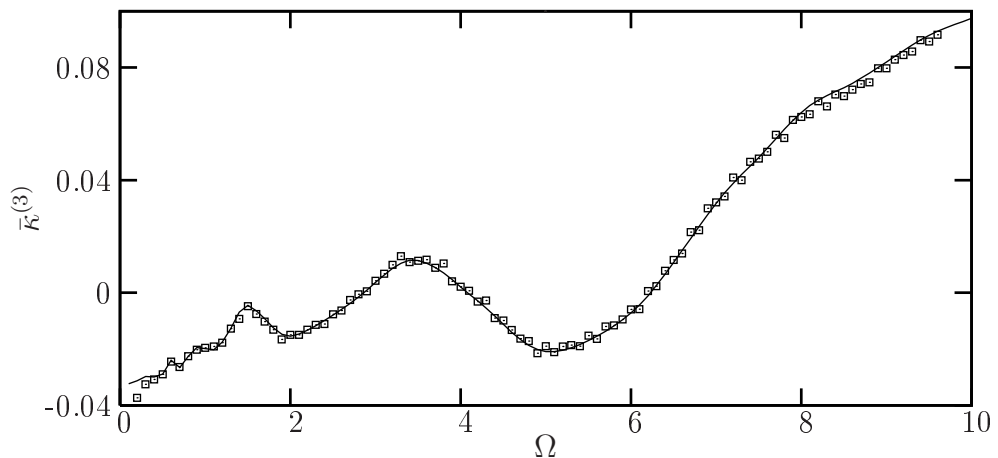

Figure 6: Comparison of $\bar{\kappa}^{(3)}=\frac{1}{\mathcal{T}} \int_{0}^{\mathcal{T}} d t \kappa^{(3)}(t)$, where $\mathcal{T}=2 \pi / \Omega$ is the period of the signal, for the toy model eq. (32) with $T_{0}=0.5, T_{1}=1.5$ and $\gamma=1.7$. The solid lines are results of the theory eq. (43) numerically evaluated according to eq. (44) truncated to 20 coefficients, while the symbols are obtained from direct simulations of the driven renewal process. Both results agree very well.

stable fixed point (rest state), which can be well modeled by a rate process, followed by a long excursion in phase space, which constitutes the excited and refractory state. This excursion takes some time which is distributed according to a waiting time distribution. As the excitation step is much more sensible to the periodic driving than the time spent on the excitation loop, we may assume this waiting time distribution $\tilde{w}(\tau)$ to be independent on the running time. The rate $\gamma(t)$ of the excitation step however, depends on the periodic signal and thus periodically on the time $t$.

In this model we have defined a phase in order to investigate synchronization between the signal and the system's dynamics. This phase was chosen to increase by $2 \pi$ each time the system has been excited and returned back to the rest state. We have derived expressions for the mean phase velocity $\omega(t)$ and effective phase diffusion constant $D(t)$. In the following we show that these expressions agree with the corresponding expressions of our general theory presented here. Namely by choosing $\mathfrak{L}=2 \pi$ the phase velocity $\omega(t)$ can be identified with $\kappa^{(1)}(t)$ while the effective phase diffusion coefficient $D(t)$ in this prior work differed from our definition of $\kappa^{(2)}(t)$ by a factor of 2 , i.e. $\kappa^{(2)}(t) \equiv 2 D(t)$.

In this two state model the mean phase velocity was given by

$$
\omega(t)=2 \pi \gamma(t) q_{2}^{(0)}(t)
$$


where the dynamics of $q_{2}^{(0)}(t)$ are governed by

$$
1-q_{2}^{(0)}(t)=\int_{0}^{\infty} d \tau \gamma(t-\tau) q_{2}^{(0)}(t-\tau) \tilde{z}(\tau)
$$

The effective diffusion coefficient was calculated as

$$
D(t)=-2 \pi \gamma(t) q_{2}^{(1)}(t)+\pi \omega(t)
$$

with

$$
\begin{aligned}
-2 \pi q_{2}^{(1)}(t)= & 2 \pi \int_{0}^{\infty} d \tau \tilde{z}(\tau) \gamma(t-\tau) q_{2}^{(1)}(t-\tau) \\
& +\int_{0}^{\infty} d \tau \tilde{z}(\tau) \omega(t-\tau)\left(\int_{0}^{\tau} d \tau^{\prime} \omega\left(t-\tau^{\prime}\right)-2 \pi\right) .
\end{aligned}
$$

The auxiliary variables $q_{2}^{(0)}$ and $q_{2}^{(1)}$ can be eliminated leading to

$$
\gamma(t) \int_{0}^{\infty} d \tau \omega(t-\tau) \tilde{z}(\tau)+\omega(t)=2 \pi \gamma(t)
$$

and

$$
\begin{aligned}
\gamma(t) \int_{0}^{\infty} d \tau & D(t-\tau) \tilde{z}(\tau)+D(t)= \\
& -2 \pi^{2} \gamma(t)+2 \pi \omega(t)+\gamma(t) \int_{0}^{\infty} d \tau \omega(t-\tau) \int_{0}^{\tau} d \tau^{\prime} \omega\left(t-\tau^{\prime}\right) \tilde{z}(\tau) .
\end{aligned}
$$

In the following we show that these equations for $\omega(t)$ and $D(t)$ are equivalent to our general eqs. 25) and (27) which for $\omega(t)$ and $D(t)$ read

$$
\int_{0}^{\infty} d \tau \omega(t-\tau) z(\tau, t-\tau)=2 \pi
$$

and

$$
\begin{aligned}
& \int_{0}^{\infty} d \tau D(t-\tau) z(\tau, t-\tau)= \\
& \int_{0}^{\infty} d \tau \omega(t-\tau) \int_{0}^{\tau} d \tau^{\prime} \omega\left(t-\tau+\tau^{\prime}\right) z(\tau, t-\tau)-2 \pi^{2}
\end{aligned}
$$

The waiting time between two events is given by the sum of the excitation time and the time spent on the excitation loop. The time dependent waiting time distribution for one cycle is therefore given by

$$
w(\tau, t)=\int_{0}^{\tau} d \tau^{\prime} \tilde{w}\left(\tau^{\prime}\right) \gamma(t+\tau) \exp \left(-\int_{t+\tau^{\prime}}^{t+\tau} d \tau^{\prime \prime} \gamma\left(\tau^{\prime \prime}\right)\right) .
$$


while the corresponding survival probability reads

$$
z(\tau, t)=\int_{0}^{\tau} d \tau^{\prime} \tilde{w}\left(\tau^{\prime}\right) \exp \left(-\int_{t+\tau^{\prime}}^{t+\tau} d \tau^{\prime \prime} \gamma\left(\tau^{\prime \prime}\right)\right)+\tilde{z}(\tau)
$$

where $\tilde{z}(\tau)=1-\int_{0}^{\tau} d \tau^{\prime} \tilde{w}(\tau)$ is the probability to spent a time longer than $\tau$ on the excitation loop. From these equations we obtain the relation

$$
\gamma(t) z(\tau, t-\tau)=w(\tau, t-\tau)+\tilde{z}(\tau) .
$$

Multiplying eqs. (51) and (53) by $\gamma(t)$ and replacing the time dependent survival probability $z(\tau, t-\tau)$ according to eq. (56) we obtain

$$
\gamma(t) \int_{0}^{\infty} d \tau \omega(t-\tau) \tilde{z}(\tau)+\int_{0}^{\infty} d \tau \omega(t-\tau) w(\tau, t-\tau)=2 \pi \gamma(t)
$$

and

$$
\begin{array}{r}
\gamma(t) \int_{0}^{\infty} d \tau\left[D(t-\tau)-\omega(t-\tau) \int_{0}^{\tau} d \tau^{\prime} \omega\left(t-\tau-\tau^{\prime}\right)\right] \tilde{z}(\tau)+ \\
\int_{0}^{\infty} d \tau\left[D(t-\tau)-\omega(t-\tau) \int_{0}^{\tau} d \tau^{\prime} \omega\left(t-\tau-\tau^{\prime}\right)\right] w(\tau, t-\tau)=-2 \pi^{2} \gamma(t)
\end{array}
$$

The second term on the left hand side in both equations can be further simplified. To this end we differentiate eqs. (51) and (53) with respect to $t$ (see appendix (E), using eq. (51) to simplify the time derivative of eq. (533) which results in

$$
\omega(t)=\int_{0}^{\infty} d \tau \omega(t-\tau) w(\tau, t-\tau)
$$

and

$$
D(t)=\int_{0}^{\infty} d \tau\left[D(t-\tau)-\omega(t-\tau) \int_{0}^{\tau} d \tau^{\prime} \omega\left(t-\tau-\tau^{\prime}\right)\right] w(\tau, t-\tau)+2 \pi \omega(t) .
$$

Note that the above equations can be equivalently derived from eq. (23) by performing the same procedure we have applied to eq. (22) in order to obtain eqs. (25) and (27).

Inserting finally eqs. (59) and (60) into eqs. (57) and (58), eventually leads to the two state model eqs. (49) and (50).

Thus we have shown that solutions of $D(t)$ and $\omega(t)$ of the general theory are also solutions of the two state model for excitable systems presented in [21]. As these solutions are in general unique we have shown the equivalence between the two models. In [21] these solutions were compared with simulations of the renewal process, showing total agreement within simulation precision. 


\section{F Time derivatives of integrals involving time dependent survival probabilities}

Let $w(\tau, t)$ be a time dependent waiting time distribution, i.e.

$$
w(\tau, z) \geq 0 \quad \forall \tau, t \quad \text { and } \quad \int_{0}^{\infty} d \tau w(\tau, t)=1 \quad \forall \tau .
$$

Let further

$$
z(\tau, t)=1-\int_{0}^{\tau} d \tau^{\prime} w\left(\tau^{\prime}, t\right)
$$

be the corresponding survival probability. Then for any sufficiently well behaved function $g(t)$ we have

$$
\frac{d}{d t} \int_{0}^{\infty} d \tau g(t-\tau) z(\tau, t-\tau)=g(t)-\int_{0}^{\infty} d \tau g(t-\tau) w(\tau, t-\tau) .
$$

This can be seen as follows:

$$
\begin{aligned}
\frac{d}{d t} & \int_{0}^{\infty} d \tau g(t-\tau) z(\tau, t-\tau) \\
& =\int_{0}^{\infty} d \tau\left(-\frac{d}{d \tau} g(t-\tau) z(\tau, t-\tau)+\left.g(t-\tau) \frac{d}{d \tau^{\prime}} z\left(\tau^{\prime}, t-\tau\right)\right|_{\tau^{\prime}=\tau}\right) \\
& =-\left.g(t-\tau) z(\tau, t-\tau)\right|_{\tau=0} ^{\infty}-\int_{0}^{\infty} d \tau g(t-\tau) w(\tau, t-\tau) \\
& =g(t)-\int_{0}^{\infty} d \tau g(t-\tau) w(\tau, t-\tau)
\end{aligned}
$$

where in the last step we assumed that $g(t-\tau) z(\tau, t-\tau)$ converges to zero as $\tau \rightarrow \infty$.

We further have the identity

$$
\begin{gathered}
\frac{d}{d t} \int_{0}^{\infty} d \tau g(t-\tau) \int_{0}^{\tau} d \tau^{\prime} g\left(t-\tau+\tau^{\prime}\right) z(\tau, t-\tau) \\
=\int_{0}^{\infty} d \tau\left(-\frac{d}{d \tau}\left[g(t-\tau) \int_{0}^{\tau} d \tau^{\prime} g\left(t-\tau+\tau^{\prime}\right) z(\tau, t-\tau)\right]\right. \\
\left.\quad+g(t-\tau) g(t) z(\tau, t-\tau)-g(t-\tau) \int_{0}^{\tau} d \tau^{\prime} g\left(t-\tau+\tau^{\prime}\right) w(\tau, t-\tau)\right) \\
=g(t) \int_{0}^{\infty} d \tau g(t-\tau) z(\tau, t-\tau) \\
\quad-\int_{0}^{\infty} d \tau g(t-\tau) \int_{0}^{\tau} d \tau^{\prime} g\left(t-\tau+\tau^{\prime}\right) w(\tau, t-\tau) .
\end{gathered}
$$




\section{References}

[1] D. R. Cox, Renewal Theory, Methuen, London (1962).

[2] N. G. van Kampen, Stochastic Processes in Physics and Chemistry, North-Holland, Amsterdam(1981).

[3] B. Lindner, L. Schimansky-Geier, A. Longtin, Phys. Rev. E 66, 031916 (2002)

[4] B. Lindner, L. Schimansky-Geier, Phys. Rev. E 60007270 (1999)

[5] T. Shimokawa, K. Pakdaman, T. Takahata, S. Tanabe, S. Sato, Biol. Cybern. 83 327(2000)

[6] B. Lindner, M. Kostur, and L. Schimansky-Geier, Fluct. Noise Lett. 1, R25 (2001).

[7] D. R. Cox, V. Isham, Point Porcesses, Chapman and Hall (1980)

[8] R. Benzi, A. Sutera and A. Vulpiani, J. Phys. A 14, L453 (1981)

C. Nicolis and G. Nicolis, Tellus 33, 225 (1981)

[9] A. Longtin, J. Stat. Phys. 70, 309 (1993)

[10] L. Gammaitoni, P. Hänggi, P. Jung, F. Marchesoni, Rev. Mod. Phys. 70, 233 (1998).

[11] B. McNamara, K. Wiesenfeld, Phys. Rev. A 394854 (1989)

[12] V. S. Anishchenko, A. B. Neiman, F. Moss, L. Schimansky-Geier, Phys. Usp. 427 (1999)

[13] J. Casado-Pascual, J. Gómez-Ordóñez, M. Morillo, J. Lehmann, I. Goychuk, P. Hänggi, Phys. Rev.E 71, 011101 (2005)

[14] J. A. Freund, A. Neiman and L. Schimansky-Geier, Europhys. Lett. $50,8(2000)$.

[15] L. Callenbach, P. Hänggi, S. J. Linz, J.A. Freund, L. SchimanskyGeier, Phys. Rev. E 65, 051110 (2002)

[16] I. Goychuk, P. Hänggi, Phys. Rev. Lett. 91, 070601 (2003).

[17] I. Goychuk, P. Hänggi, Phys. Rev. E 69, 021104 (2004).

[18] T. Prager, L. Schimansky-Geier, Phys. Rev. Lett. 91, 230601 (2003).

[19] R.L. Stratonovich, Topics in the Theory of Random Noise,Gordon and Breach (1963) 
[20] D.T. Gillespie, Phys. Letters 64A, 22(1977)

[21] T. Prager and L. Schimansky-Geier, Phys. Rev. E 71, 031112 (2005) 